\title{
Congenital diaphragmatic hernia: postnatal predictors of mortality
}

\author{
Gisela L. Salas, M.D. ${ }^{a}$, Jesica C. Otaño, M.D. ${ }^{a}$, Claudia M. Cannizzaro, M.D. ${ }^{a}$,
} María T. Mazzucchelli, M.D. ${ }^{a}$ and Gustavo S. Goldsmit, M.D. ${ }^{a}$

\begin{abstract}
Congenital diaphragmatic hernia (CDH) prevalence is low while its associated morbidity and mortality rates are high. Postnatal prognostic factors on the first day of life are useful for predicting the outcome.

Objectives. To determine the mortality predictive ability of postnatal echocardiographic, clinical, and biochemical factors among newborn infants with $\mathrm{CDH}$ in their first day of life.

Method. Observational analytical study of a retrospective cohort. Patients with $\mathrm{CDH}$ were consecutively included between March 2012 and November 2018. On the first day of life, analyzed predictors were the oxygenation index (OI), the highest partial pressure of carbon dioxide $\left(\mathrm{pCO}_{2}\right)$ level in blood, the SNAPPE II severity score, the echocardiography, and the $\mathrm{N}$-terminal pro-B-type natriuretic peptide (NTproBNP) value.

Results. The population consisted of 178 patients with CDH. Survival was $75 \%$. Extracorporeal membrane oxygenation was used in $24 \%$. The early onset of systemic or suprasystemic pulmonary hypertension showed no predictive ability (OR: 2.2, $95 \%$ CI: 0.8-8), $p=0.1$. NT-proBNP did not show good discrimination either (area under the curve [AUC]:0.46, $p=0.67$ ). The OI, SNAPPE II score, and the highest $\mathrm{pCO}_{2}$ level showed adequate discrimination power, AUC for OI: 0.82, AUC for SNAPPE II: 0.86, and AUC for $\mathrm{pCO}_{2}: 0.75, p<0.001$.

Conclusion. The SNAPPE II score, the OI, and the highest $\mathrm{pCO}_{2}$ level measured on the first day of life, showed a good predictive ability in terms of the course of the disease; the SNAPPE II score was better than the OI and the highest $\mathrm{pCO}_{2}$ level. Key words: diaphragmatic hernia, mortality, determinants.
\end{abstract}

Neonatal Intensive Care Unit, Hospital de Pediatría SAMIC "Prof. Dr. Juan

P. Garrahan", Autonomous City of Buenos Aires, Argentina.

E-mail address:

Gisela L. Salas, M.D.: salasenator@gmail.com

http: / / dx.doi.org/10.5546/ aap.2020.eng.173

To cite: Salas GL, Otaño JC, Cannizzaro CM, Mazzucchelli MT, Goldsmit GS. Congenital diaphragmatic hernia: postnatal predictors of mortality. Arch Argent Pediatr 2020;118(3):173-179.
Funding:

None.

Conflict of interest:

None.

Received: 6-6-2019

Accepted: 11-13-2019

\section{INTRODUCTION}

Congenital diaphragmatic hernia $(\mathrm{CDH})$ occurs in approximately 1 in every 3000-5000 live births and has high perinatal morbidity and mortality rates. $\mathrm{CDH}$ is characterized by the presence of pulmonary hypoplasia or agenesis involving the left, right or both lungs, the failure of diaphragmatic closure, and the herniation of abdominal organs into the thoracic cavity. ${ }^{1,2}$ Morbidity and mortality are especially determined by the association with other malformations, the degree of pulmonary hypoplasia and the presence of secondary pulmonary hypertension. ${ }^{3}$

The etiopathogenesis of $\mathrm{CDH}$ is unknown. In the beginning, it was believed that the defect was associated with failure of diaphragmatic closure. At present, it is postulated that a disruption occurs during lung development, as a first step towards the development of $\mathrm{CDH}$, followed by failure of diaphragmatic closure. ${ }^{4}$

In recent years, the survival of patients with $\mathrm{CDH}$ has improved dramatically and has ranged between $50 \%$ and $90 \%$, depending on the units. However, this is accompanied by a higher long-term morbidity. ${ }^{5}$

The availability of antenatal diagnosis may vary among different countries. It is $70 \%$ according to world reports on $\mathrm{CDH}$ (Congenital Diaphragmatic Hernia [CDH] Study Group). ${ }^{6}$ The use of prenatal ultrasound predictors, the observed-to-expected lung-to-head ratio (O/E LHR), extent of liver herniation into the thoracic cavity, fetal echocardiographies, ${ }^{7,8}$ and the observed-to-expected total fetal lung volume (O/E TFLV) determined by magnetic resonance imaging (MRI), 
are very good antenatal predictors of the outcome of patients with $\mathrm{CDH} .{ }^{9,10}$

However, in those regions where antenatal diagnosis rates are lower than those reported internationally and the availability of resources, such as fetal MRI and the estimation of ultrasound predictors, is limited, postnatal risk factors indicative of a poor outcome become relevant.

The Score for Neonatal Acute Physiology with Perinatal Extension-II (SNAPPE II), developed by Richardson and modified in 1998, is a severity score used to classify the risk of all patients admitted to a neonatal intensive care unit. The SNAPPE II score has been widely accepted because it is simple, easy to record, and has an adequate predictive ability. ${ }^{11}$

The B-type natriuretic peptide (BNP) and the $\mathrm{N}$-terminal pro-B-type natriuretic peptide (NTproBNP) are biomarkers released into the circulation in response to ventricular volume or pressure overload. As a prognostic factor, it has been widely used in patients of all age groups, especially in those with cardiac involvement and pulmonary hypertension. ${ }^{12}$

An echocardiography in patients with $\mathrm{CDH}$ in their first day of life helps to estimate the degree of pulmonary hypertension. The presence of suprasystemic pulmonary hypertension has been considered a risk factor for mortality.$^{13}$ It is also worth mentioning the prognostic value of measuring blood gases and the oxygenation index $(\mathrm{OI}){ }^{14}$

The objective of this study was to determine the mortality predictive ability of postnatal echocardiographic, clinical and biochemical factors among newborn infants with $\mathrm{CDH}$ measured in their first day of life.

\section{MATERIAL AND METHODS}

This was an observational analytical study of a retrospective cohort. All patients with $\mathrm{CDH}$ admitted to the Neonatal Intensive Care Unit of Hospital de Pediatría "Prof. Dr. Juan P. Garrahan", level IIIlb, were consecutively included between March 2012 and November 2018. Patients admitted after their first day of life were excluded.

Predictors of mortality analyzed included demographic outcome measures, the OI, highest value of partial pressure of carbon dioxide in blood $\left(\mathrm{pCO}_{2}\right)$, the SNAPPE II severity score, the degree of pulmonary hypertension, presence of ventricular dysfunction by echocardiography, and the NT-proBNP level, all measured on the first day of life. Demographic outcome measures included gestational age, birth weight, sex, the presence of congenital heart disease, antenatal diagnosis, hernia laterality, referred patients, mode of delivery, and chromosomal disorder.

The presence of a congenital heart disease was diagnosed by fetal and / or postnatal echocardiography. Congenital heart diseases were defined as those requiring surgical repair. Small or restrictive atrial and/or ventricular septal defects and the presence of a patent ductus arteriosus were excluded, even if they required surgical repair.

OI was estimated based on the following formula: mean arterial pressure (MAP) $x$ fraction of inspired oxygen $\left(\mathrm{FiO}_{2}\right)$ /postductal partial pressure of oxygen $\left(\mathrm{paO}_{2}\right)$. The SNAPPE II score assigns different scores based on the following outcome measures: birth weight, blood pressure, body temperature, blood $\mathrm{pH}$, urine output, Apgar score at 5 minutes, $\mathrm{paO}_{2} / \mathrm{FiO}_{2^{\prime}}$ occurrence of seizures and intrauterine growth restriction. ${ }^{11}$

The highest $\mathrm{pCO}_{2}$ value was determined by selecting, among blood gases tests performed on the first day of life, the one showing the highest level of $\mathrm{pCO}_{2}$. In all cases, blood gases were analyzed in samples collected from catheters inserted in the umbilical or femoral artery, and these were postductal measurements.

NT-proBNP levels were obtained using 1-mL blood samples collected from the arterial catheter in a sodium heparin tube and then centrifuged. Measurements were made by microparticle enzyme immunoassay (AXSYM system, ABBOTT Laboratories), and values were reported as $\mathrm{pg} / \mathrm{mL}$.

The echocardiography was performed on newborns within the first 24 hours of life by the hospital cardiologists. The echocardiographic outcome measures analyzed included the degree of pulmonary hypertension, classified as infrasystemic, systemic or suprasystemic, and the presence of ventricular dysfunction.

The study was approved by the Research and Teaching Board of Hospital de Pediatría "Prof. Dr. Juan P. Garrahan".

Statistical analysis: All outcome measures were summarized using descriptive statistics of central tendency, position, and dispersion. Numerical outcome measures were compared using a Wilcoxon non-parametric test or t test, based on their distribution. Categorical outcome measures were compared using the $\chi^{2}$ test. Nonparametric receiver operating characteristic (ROC) curves were developed. The area under the curve (AUC) and the $95 \%$ confidence 
interval (CI), sensitivity (S), specificity (Sp), positive likelihood ratio ( $\mathrm{LR}+$ ), and negative likelihood ratio (LR-) were estimated for each cutoff value. The marker's cut-off value was defined as the one that reached the maximum sensitivity and specificity. Based on the selected cut-off value, the outcome measure was dichotomized and the relative risk (RR) and its corresponding $95 \%$ CI were estimated. The odds ratio (OR) and its corresponding $95 \%$ CI were estimated for echocardiographic outcome measures. A value of $p<0.05$ was considered statistically significant. The statistical analysis was performed with the STATA SE 12.0 software (StataCorp LP, USA).

\section{RESULTS}

The study population consisted of 182 patients with $\mathrm{CDH}$. Four patients were excluded because they were admitted to the unit after the first day of life. A total of 178 patients were analyzed. The rate of overall survival was $75 \%$. Table 1 describes the clinical and demographic characteristics of the study population. Figure 1 shows the distribution of $\mathrm{CDH}$ by year and survival per year.

Demographic and clinical outcome measures were compared based on mortality and are shown in Table 2. The outcome measures that showed a statistically significant difference in terms of mortality were the gestational age and the presence of congenital heart disease.

TABLE 1. General characteristics of the study population

\begin{tabular}{lc}
\hline & CDH \\
$\mathbf{n}=\mathbf{1 7 8}$
\end{tabular}

${ }^{1}$ Values are described as mean and standard deviation (SD) and

${ }^{2}$ as median and interquartile range (IQR).

$\mathrm{CDH}$ : congenital diaphragmatic hernia.

SD: standard deviation.

ECMO: extracorporeal membrane oxygenation.

FIGURE 1. Total number of patients with congenital diaphragmatic hernia and total number of survivors at the time of discharge, by year of admission to the Neonatal Intensive Care Unit of Hospital de Pediatrí "Prof. Dr. Juan P. Garrahan"

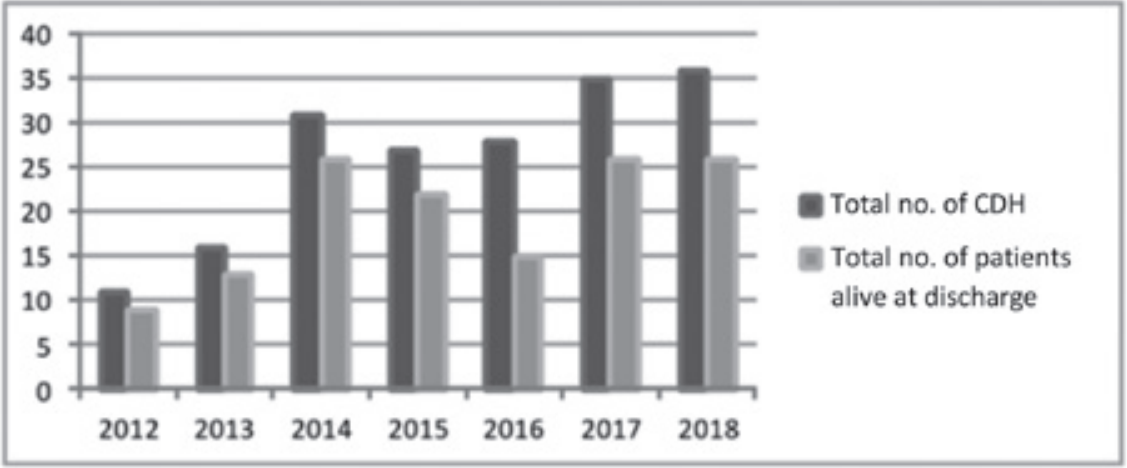

$\mathrm{CDH}$ : congenital diaphragmatic hernia. 
NT-proBNP levels showed a wide dispersion (Table 2), but there was no statistically significant difference between both groups $(p=0.67)$. The ROC curve described the poor discrimination, an AUC of $0.46, p=0.67$ (Figure 2).

Systemic or suprasystemic pulmonary hypertension occurred in $75 \%$ of survivors and in $83 \%$ of deceased patients, OR: 2.2 (95\% CI: 0.8-8), $p=0.1$. This was not a risk factor for mortality. Also, the presence of ventricular dysfunction was not a risk factor, OR: 2.3 (95\% CI: 0.8-6), $p=0.05$.

The outcome measures that showed a statistically significant difference between both groups were SNAPPE II score, OI, and the highest level of $\mathrm{pCO}_{2}$, measured on the first day of life. SNAPPE II score showed an adequate discrimination, an AUC of 0.86 (95\% CI: 0.780.93), cut-off point: 29 (S: $80 \%$; Sp: $81 \%$; LR+: 3.7; LR-: 0.15). After dichotomizing the sample into two groups, SNAPPE II score $<29$ or $\geq 29$, the RR was 9.3 (95\% CI: 3.9-22), $p=<0.001$. Patients with CDH who, on their first day of life, had a SNAPPE II score $\geq 29$ had a risk of death 9.3 times higher than those with a SNAPPE II score $<29$ (Figure 2).

The OI showed an adequate discrimination, with an AUC of 0.82 (95\% CI: 0.75-0.88), cut-off point: 18 (S: $71 \%$; Sp: $85 \%$; LHR+: 7.3; LHR-: 0.3). The OI was dichotomized based on the cut-off point of 18, RR: 4.6 (95\% CI: 2.6-8), $p=<0.001$. Patients with $\mathrm{CDH}$ who, on their first day of life, had an $\mathrm{OI} \geq 18$ had a risk of death 4.6 times higher than those with an $\mathrm{OI}<18$ (Figure 2).

The highest level of $\mathrm{pCO}_{2}$ also showed an adequate discrimination, with an AUC of
0.75 (95\% CI: 0.68-0.81), cut-off point: 60 (S: $61 \%$; Sp: $81 \%$; LHR+: 2.7; LHR-: 0.5). After dichotomizing the $\mathrm{pCO}_{2}$ based on the cut-off point, the RR was 3 (95\% CI: 1.8-4.8), $p=<0.001$. Patients who, on their first day of life, had a $\mathrm{pCO}_{2}$ $\geq 60 \mathrm{mmHg}$ had a risk of death 3 times higher than those with a $\mathrm{pCO}_{2}<60 \mathrm{mmHg}$ (Figure 2).

\section{DISCUSSION}

The results observed in this study show that the SNAPPE II score, OI, and the highest level of $\mathrm{pCO}_{2}$ in blood, measured on the first day of life, were predictors of mortality and had a good discrimination power. Such benefit was not demonstrated by the NT-proBNP levels or the presence of systemic or suprasystemic pulmonary hypertension and/or ventricular dysfunction, measured by echocardiography. In this study, the demographic outcome measures that showed a statistically significant difference were gestational age and presence of congenital heart disease.

Evidence has demonstrated that a $\mathrm{CDH}$ in association with prematurity increases mortality. An analysis of the CDH Study Group database reported a $73 \%$ survival in patients with a $\mathrm{CDH}$ with a gestational age of at least 37 weeks compared to a $53 \%$ survival in preterm infants.

Congenital heart disease was observed in 8 patients, with an incidence of $4.5 \%$, lower than the $8 \%$ described in international reports. ${ }^{16}$ Only 2 patients with congenital heart disease lived to undergo a surgical repair and survived after discharge; the remaining 6 patients died before being subjected to a cardiac surgical repair.

The presence of chromosomal abnormalities

TABLE 2. Comparison of demographic and clinical outcome measures based on survival

\begin{tabular}{|c|c|c|c|}
\hline & $\begin{array}{l}\text { CDH patients alive at discharge } \\
\qquad \mathrm{n}=134\end{array}$ & $\begin{array}{l}\text { CDH patients who died } \\
\qquad \mathrm{n}=44\end{array}$ & $p$ \\
\hline 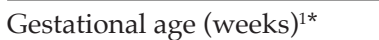 & $37.4($ SD 1.3$)$ & $36.5(\mathrm{SD} 2.1)$ & 0.03 \\
\hline Male gender $(\%)^{3}$ & 56 & 59 & 0.6 \\
\hline Birth weight $(\mathrm{g})^{1 *}$ & 2974 (SD 472) & 2888 (SD 545) & 0.3 \\
\hline Congenital heart disease $(\%)^{3}$ & 1.5 & 13.6 & 0.0000 \\
\hline Chromosome disorder $(\%)^{3}$ & 3.7 & 4.5 & 0.16 \\
\hline Antenatal diagnosis $(\%)^{3}$ & 70 & 80 & 0.05 \\
\hline Referred patients $(\%)^{3}$ & 56 & 43 & 0.1 \\
\hline Left-sided $(\%)^{3}$ & 88 & 82 & 0.3 \\
\hline NT-proBNP ${ }^{2 * *}$ & $8051(2200-13000)$ & $8700(6200-11000)$ & 0.67 \\
\hline SNAPPE II score ${ }^{2 * *}$ & $13.0(5-23)$ & $48.6(32-62)$ & 0.0000 \\
\hline $\mathrm{OI}^{2 * \star}$ & $6(4-13)$ & $27(12-46)$ & 0.0000 \\
\hline High $\mathrm{pCO}_{2}^{2 * *}$ & $48(41-59)$ & $66(64.5-79.3)$ & 0.0000 \\
\hline
\end{tabular}

${ }^{1}$ T test. ${ }^{2}$ Wilcoxon test. ${ }^{3} \chi^{2}$ test. NT-proBNP: N-terminal pro-B-type natriuretic peptide.

OI: oxygenation index. $\mathrm{pCO}_{2}$ : partial pressure of carbon dioxide in blood.

* Values are described as mean and standard deviation (SD). ** Median and interquartile range. 
was not associated with a significant difference between alive and dead patients. Multicenter studies have used the association between $\mathrm{CDH}$ and chromosomal abnormalities as part of a risk score for $\mathrm{CDH} .{ }^{17}$ Although the incidence of chromosomal disorders in the studied population is low, chromosomal testing is deficient both in the population of patients with an antenatal and postnatal diagnosis of $\mathrm{CDH}$.

In this study, SNAPPE II score showed an excellent discrimination power. Other authors have also assessed this scoring system in $\mathrm{CDH}$. Chiu et al. reported that the SNAPPE II score was an excellent predictor of mortality in $\mathrm{CDH}$; survivors had a score of 20 (standard deviation [SD]: 15), whereas dead patients had a score of 41 (SD: 16) $(p=0.001) .{ }^{18}$

NT-proBNP has been studied in the neonatal population, mostly among patients with patent ductus arteriosus, pulmonary hypertension, and congenital heart diseases. ${ }^{12}$ It shows a high prognostic value when serial samples are collected; however, an isolated value provides poor discrimination. ${ }^{19}$ In this study, NT-proBNP levels on the first day of life were not correlated to mortality and had a wide dispersion. This was not consistent with the study carried out by Steurer et al., where the BNP level showed an excellent discrimination on the first day of life of patients with $\mathrm{CDH} .{ }^{20}$ Such difference with our population may be due to the $\mathrm{N}$-terminal peptide (NT-proBNP) measurement, which is more stable and has a longer half-life than the BNP.

The degree of pulmonary hypertension assessed by echocardiography on the first day of life did not display a discrimination power in relation to mortality among these patients in this study. More than $70 \%$ of patients had systemic or suprasystemic pulmonary hypertension, but this was not correlated to survival, probably due to the transitional hemodynamic changes that take place during the first day of life. Other studies agree that the degree of pulmonary hypertension measured by echocardiography at the first week

FIGURE 2. Receiver operating characteristic curve showing the discrimination ability of 1: NT-proBNP, 2: oxygenation index, 3: SNAPPE II score, and 4: highest $\mathrm{CCO}_{2}$ level in arterial blood to predict mortality in patients with congenital diaphragmatic hernia

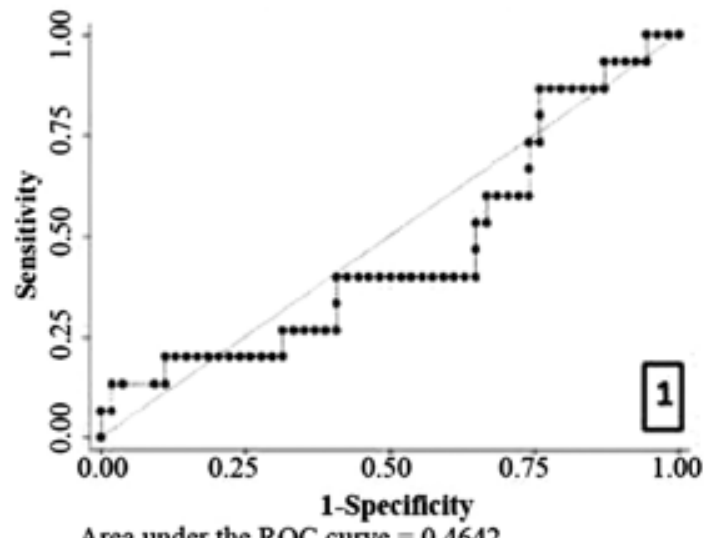

Area under the ROC curve $=0.4642$

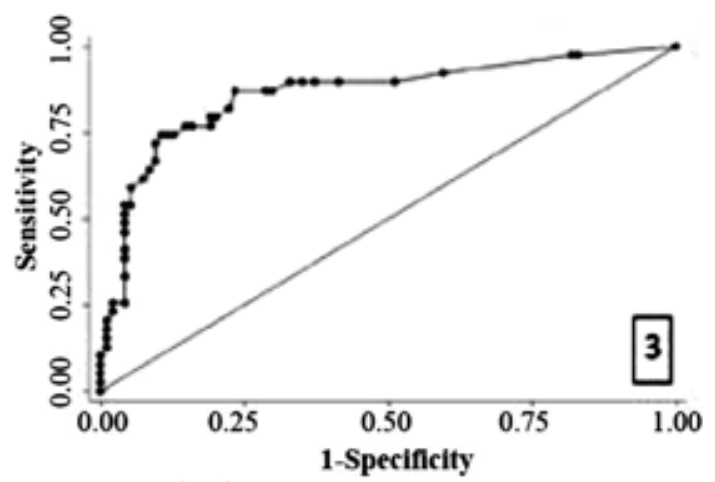

Area under the ROC curve $=0.8622$

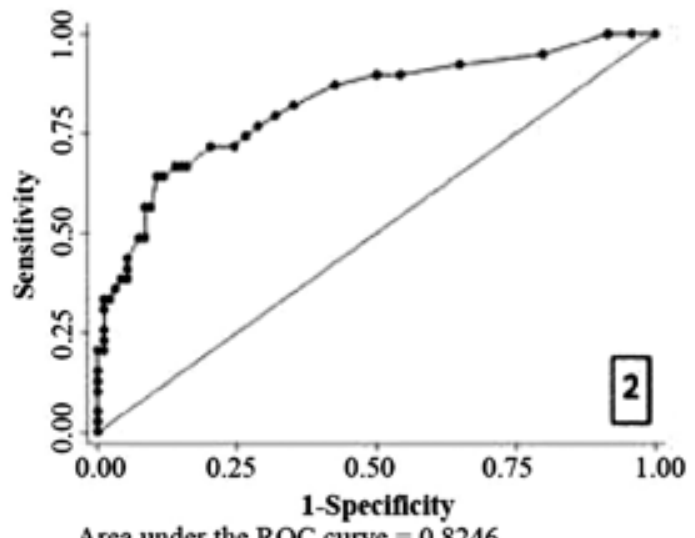

Area under the ROC curve $=0.8246$

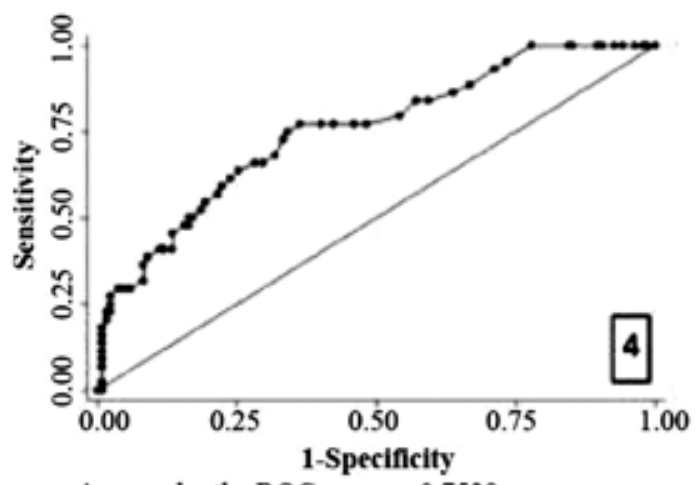

Area under the ROC curve $=0.7539$

ROC: receiver operating characteristic. 
of life is a better predictor of outcome than when measured on the first day of life..$^{20}$ The extent of ventricular dysfunction is a better prognostic predictor on the first day of life. ${ }^{21}$

In our study, a major difference was observed in relation to ventricular dysfunction on the first day of life; $67 \%$ of survivors had ventricular dysfunction versus $83 \%$ of those who died. Although such difference is not significant, it describes a trend towards a prediction of mortality, which would probably become more evident if more patients had been included in this cohort.

The OI and highest level of $\mathrm{pCO}_{2}$ in blood have been considered markers of lung development. It has been estimated that, in patients with $\mathrm{CDH}$, the $\mathrm{pCO}_{2}$ level is better than $\mathrm{paO}_{2}$ to predict pulmonary hypoplasia degree because oxygenation may be affected by the degree of pulmonary hypertension at the time of sample collection. ${ }^{22,23}$

The levels of $\mathrm{pCO}_{2}$ in blood may be used to estimate the degree of pulmonary hypoplasia. An increase in $\mathrm{pCO}_{2}$ levels is usually associated with high frequency ventilation (HFV) requirements on the first day of life. This ventilation mode in patients with $\mathrm{CDH}$ may induce greater ventricular dysfunction and no benefits have been shown in comparison with conventional ventilation. ${ }^{24}$ The development of scoring systems that combine antenatal and postnatal risk factors in patients with CDH may improve the early predictive ability to assess the risk of a poor outcome in this population. ${ }^{7,25}$

In relation to the weaknesses of this study, it is worth mentioning that this is a retrospective study conducted at a single site. From a methodological perspective, the RR of SNAPPE II, $\mathrm{OI}$, and highest $\mathrm{pCO}_{2}$ outcome measures was not adjusted for other confounders and was, instead, analyzed independently. The lack of classification of ventricular dysfunction by echocardiography into right, left and/or biventricular is a major limitation because the current bibliography describes the presence of left ventricular dysfunction in these patients as a strong predictor of outcome. ${ }^{21,26}$ The strengths of this study are the large number of patients studied, considering that this is a rare condition, and that it was conducted at a referral hospital, with a high survival rate $(75 \%)$, whereas recent regional studies have reported a survival rate by $32 \% .{ }^{27}$

\section{CONCLUSION}

SNAPPE II score, OI, and the highest level of $\mathrm{pCO}_{2}$ in blood, measured on the first day of life among patients with $\mathrm{CDH}$, showed an adequate predictive ability related to the outcome. SNAPPE II score had a better performance than $\mathrm{OI}$ and the highest level of $\mathrm{pCO}_{2}$.

\section{Acknowledgments}

The authors would like to thank Susana Patricia Rodríguez, M.D. and Director of Research and Teaching Board of Hospital de Pediatría "Prof. Dr. Juan P. Garrahan", for the valuable collaboration in the development of this study.

\section{REFERENCES}

1. Kluth D, Tenbrinck R, Von Ekesparre M, Kangah R, et al. The natural history of congenital diaphragmatic hernia and pulmonary hypoplasia in the embryo. J Pediatr Surg. 1993; 28(3):456-62.

2. Eastwood MP, Russo FM, Toelen J, Deprest J. Medical interventions to reverse pulmonary hypoplasia in the animal model of congenital diaphragmatic hernia: A systematic review. Pediatr Pulmonol. 2015; 50(8):820-38.

3. Harting MT. Congenital diaphragmatic hernia-associated pulmonary hypertension. Semin Pediatr Surg. 2017; 26(3):147-53.

4. Keijzer R, Liu J, Deimling J, Tibboel D, et al. Dual-hit hypothesis explains pulmonary hypoplasia in the nitrofen model of congenital diaphragmatic hernia. Am J Pathol. 2000; 156(4):1299-306.

5. Wynn J, Krishnan U, Aspelund G, Zhang Y, et al. Outcomes of congenital diaphragmatic hernia in the modern era of management. J Pediatr. 2013; 163(1):114-9.e1.

6. Coughlin MA, Werner NL, Gajarski R, Gadepalli S, et al. Prenatally diagnosed severe CDH: mortality and morbidity remain high. J Pediatr Surg. 2016; 51(7):1091-5.

7. Werner NL, Coughlin M, Kunisaki SM, Hirschl R, et al. Prenatal and postnatal markers of severity in congenital diaphragmatichernia have similar prognostic ability. Prenat Diagn. 2016; 36(2):107-11.

8. Kastenholz KE, Weis M, Hagelstein C, Weiss C, et al. Correlation of observed-to-expected MRI fetal lung volume and ultrasound lung-to-head ratio at different gestational times in fetuses with congenital diaphragmatic hernia. AJR Am J Roentgenol. 2016; 206(4):856-66.

9. Kilian AK, Schaible T, Hofmann V, Brade J, et al. Congenital diaphragmatic hernia: predictive value of MRI relative lung-to-head ratio compared with MRI fetal lung volume and sonographic lung-to-head ratio. AJR Am J Roentgenol. 2009; 192(1):153-8.

10. Bebbington M, Victoria T, Danzer E, Moldenhauer J, et al. Comparison of ultrasound and magnetic resonance imaging parameters in predicting survival in isolated leftsided congenital diaphragmatic hernia. Ultrasound Obstet Gynecol. 2014; 43(6):670-4.

11. Richardson D, Corcoran J, Escobar G, Lee SK. SNAP-II and SNAPPE-II: Simplified newborn illness severity and mortality risk scores. J Pediatr. 2001; 138(1):92-100.

12. El-Khuffash A, Molloy EJ. Are B-type natriuretic peptide (BNP) and N-terminal-pro-BNP useful in neonates? Arch Dis Child Fetal Neonatal Ed. 2007; 92(4):F320-4.

13. Suda K, Bigras JL, Bohn D, Hornberger LK, et al. Echocardiographic predictors of outcome in newborns 
with congenital diaphragmatic hernia. Pediatrics. 2000; 105(5):1106-9.

14. Tan YW, Ali K, Andradi G, Sasidharan L, et al. Prognostic value of the oxygenation index to predict survival and timing of surgery in infants with congenital diaphragmatic hernia. J Pediatr Surg. 2019; 54(8):1567-72.

15. Tsao K, Allison N, Harting MT, Lally PA, et al. Congenital diaphragmatic hernia in the preterm infant. Surgery. 2010; 148(2):404-10.

16. Patel M, Bell C, Lally K, Lally $\mathrm{P}$, et al. Lowest PaCO2 on the first day of life predicts mortality and morbidity among infants with congenital diaphragmatic hernia. J Perinatol. 2019: 39(2):229-36.

17. Brindle ME, Cook EF, Tibboel D, Lally P, et al. A clinical prediction rule for the severity of congenital diaphragmatic hernias in newborns. Pediatrics. 2014; 134(2):e413-9.

18. Chiu L, Desai J, Shanti C, Rane S, et al. SNAPPE II Score As a Predictor of Survival in Neonates with Congenital Diaphragmatic Hernia: A Single Center Experience. Eur J Pediatr Surg. 2016; 26(04):316-21.

19. Salas G, Josefkowicz M, Goldsmit G, Disa G, et al. Péptido natriurético tipo $\mathrm{B}$ : utilidad en el manejo de recién nacidos críticamente enfermos. Arch Argent Pediatr. 2017; 115(5):483-9.

20. Steurer M, Moon-Grady A, Fineman J, Sun C, et al. B-type natriuretic peptide: a prognostic marker in congenital diaphragmatic hernia. Pediatr Res. 2014; 76(6):549-54.

21. Patel N, Massolo A, Paria A, Stenhouse E, et al. Early postnatal ventricular dysfunction is associated with disease severity in patients with congenital diaphragmatic hernia. J Pediatr. 2018; 203:400-7.e1.

22. Grizelj R, Bojanić K, Pritišanac E, Luetic T, et al. Survival prediction of high-risk outborn neonates with congenital diaphragmatic hernia from capillary blood gases. BMC Pediatr. 2016; 16:114.

23. Schultz CM, DiGeronimo RJ, Yoder BA. Congenital diaphragmatic hernia: a simplified postnatal predictor of outcome. J Pediatr Surg. 2007; 42(3):510-6.

24. Van den Hout L, TibboelD, VijfhuizeS, Te Beest H, et al. The VICI-trial: high frequency oscillation versus conventional mechanical ventilation in newborns with congenital diaphragmatic hernia: an international multicentre randomized controlled trial. BMC Pediatr. 2011; 11:98.

25. Oh C, Youn JK, Han J, Yang H, et al. Predicting Survival of Congenital Diaphragmatic Hernia on the First Day of Life. World J Surg. 2019; 43(1):282-90.

26. KinsellaJP,SteinhornRH,MullenMP,HopperR,etal.TheLeft Ventriclein Congenital DiaphragmaticHernia:Implications for the Management of Pulmonary Hypertension. J Pediatr. 2018; 197:17-22.

27. Cruz-Martínez R, Etchegaray A, Molina-Giraldo S, NietoCastro B, et al. A multicentre study to predict neonatal survival according to lung-to-head ratio and liver herniation in fetuses with left congenital diaphragmatichernia (CDH): Hidden mortality from the Latin American CDH Study Group Registry. Prenat Diagn. 2019; 39(7):519-26. 